\title{
Morphological comparison between Doryteuthis pleii and D. sanpaulensis (Cephalopoda, Myopsida, Loliginidae) from Brazil
}

\author{
Paulo Presti Migliavacca ${ }^{1,2}$ \& Luiz Ricardo L. Simone ${ }^{1,3}$ \\ 1 Universidade de São Paulo (USP), Museu de Zoologia (MZUSP). São Paulo, SP, Brasil. \\ 2 ORCID: http://orcid.org/0000-0003-3803-6028. E-mail: paulo.migliavacca@usp.br \\ ${ }^{3}$ ORCID: http://orcid.org/0000-0002-1397-9823. E-mail: Irsimone@usp.br
}

\begin{abstract}
The distinction of squid species in the genus Doryteuthis is not easy due to their morphological similarity, lack of conspicuous specific characters, and overlap geographical occurrence. This difficulty has leading to an almost exclusive molecular approach, and a premature neglect of the morpho-anatomy. To emphasize that the squid phenotypic features can be useful to identify, as well as to perform any comparative analyses (such as taxonomy and phylogeny), two close species were selected as outset. Doryteuthis pleii and D. sanpaulensis are common sympatric squids in Brazilian waters, commonly used in fisheries, not so difficult to distinguish by external features of the adult specimens. The samples were analyzed from biometric data to dissections, and the found most expressive characters to distinguish them are the mantle-fin ratio; morphology of the tentacle club, its ratio compared to the mantle length; hectocotylus morphology and nidamental gland morphology.
\end{abstract}

Key-Words. Anatomy; Characters; Squid; Sympatric; Taxonomy.

\section{INTRODUCTION}

Loliginidae is a widely distributed squid family of economic importance, extensively targeted as fishery products and with a relevant role in an intricate marine food web (Fields, 1965; Anderson, 2000; Vecchione \& Young, 2010a; GranadosAmores et al., 2014). It comprises 47 species distributed in 10 genera, all of which are pelagic (Jereb \& Roper, 2010; Vecchione \& Young, 2010a; Granados-Amores et al., 2014). Loliginids usually have the posterior end of the fins connected to the mantle and four rows of suckers on the tentacular club [except for Pickfordiateuthis (Voss, 1953) (see Brakoniecki, 1996; Vecchione \& Young, 2010a)]. Initially, two of the commonest species in Brazilian coast, Doryteuthis pleii (Blainville, 1823) and D. sanpaulensis (Brakoniecki, 1984), were placed in the genus Loligo, a genus that is now restricted to the Eastern Atlantic (Vecchione et al., 2005).

There is eight species of Doryteuthis, which are sometimes misidentified due to their morphological similarity, the lack of conspicuous, indubitable specific characters to define them, and their overlapped geographical distribution (Vecchione et al., 1998; Vecchione \& Young, 2010b).
Nowadays, detailed studies on squid anatomy are scarce, even though morphological data have proved to be very useful in producing phylogenetic hypotheses and defining relationships within mollusk groups (e.g., Simone, 2011; Couto et al., 2015; Dornellas \& Simone, 2015). There are more recent papers about molecular analyses than morphological studies. Still, most morphological studies on squids are focused on external characters, barely taking into account the internal anatomy.

Therefore, considering that D. pleii and D. sanpaulensis are relatively locally common, have economic importance in Brazilian waters, and are sympatric, the main purpose of this study is to clarify the differences between these two species, which are commonly misidentified, focusing on both internal and external characters, a more practical approach to determine the species from laboratories to fishmongers. It is not the intention of this paper to perform any statistical analysis, an approach already done elsewhere (Juanicó, 1979), focusing, here, the morpho-anatomical features only. The pioneer study by Juanicó (1979) is commonly used to base the identification of $D$. pleii and $D$. sanpaulensis (then called Loligo brasiliensis Blainville, 1823), and has a comprehensive morphometric analysis of the external features and of the gladius. 
However, a detailed comparison of the internal anatomy of both species is still inedited, a gap fulfilled herein.

\section{MATERIAL AND METHODS}

The material examined belongs to the Malacological Collection of the Museu de Zoologia da Universidade deSão Paulo (MZSP). Specimens preserved in $70 \%$ ethanol were measured following the standards of ENREF_4, Roper \& Voss (1983), Laptikhovsky et al. (2009) and Vecchione et al. (2015) and dissected through usual procedures.

Biometric data were clustered in a table and only the mantle length (ML), fin length ( $F L)$, arms length (Al, All, Alll, AIV - counted dorsally to ventrally), eye diameter (ED) and hectocotylus length $(\mathrm{HtL})$ were used in order to describe and compare the species. Some morphological characters (e.g., tentacle length) are prone to alterations during fixation and therefore were not listed here (Vecchione et al., 2015). All the arms were measured and average values were taken for each arm pair. Some measurements were converted to percentage of the mantle length (after Vecchione et al., 2015) in order to standardize the values, allowing for the comparison between $D$. pleii and D. sanpaulensis. In total, 40 specimens of each species were measured. Drawings were made with the aid of a camera lucida. Radulae of both species were manually extracted and prepared by immersion in $\mathrm{KOH}$ and further ultrasonic cleaning, followed by immersion in distilled water, prior to Scanning Electron Microscope (SEM) examination.

Abbreviations in the figures: aa, anterior aorta; ad, adductor muscle; ag, accessory nidamental gland; ah, anterior head retractor nerve; am, anterior mass; an, anus; ap, posterior aorta; ar, arm; at, aortic trunk; ba, branchial artery; bh, branchial heart; bl, blood vessel; bm, buccal bulb; br, branchial muscle; bv, branchial vein; ca, caecum; cg, capillaries of the gill; cn, collar nerve; es, esophagus; ey, eye; fi, fin; fn, funnel; fu, mantle-funnel locking apparatus; gi, gill; gs, stellate ganglion; he, head; ib, interbrachial commissure; ik, ink sac; ki, kidney (nephridium); Iv, lateral nephridial vessel; $\mathrm{ma}$, mantle aorta; $\mathrm{mm}$, middle mass; $\mathrm{mt}$, muscular mantle; ni, nidamental gland; ns, Needham sac; oc, inferior anterior oculomotor nerve; og, oviducal gland; ol, optic lobe; oy, ovary; pe, penis; pm, posterior mass; pn, pallial nerve; po, post-orbital nerve; pv, posterior vena cava; rt, rectum; sh, systemic heart; sk, skull; so, superior posterior oculomotor nerve; sp, spermatophoric gland; st, stomach; sy, statolith; te, tentacle; ts, testis; vg, vagina.

\section{RESULTS}

\section{Doryteuthis pleii (Blainville, 1823)}

(Figs. 1, 3, 5, 6)

\section{Synonymy see Cohen (1976). Complement:}

Loligo plei [sic]: Cohen, 1976: 341 (figs. 25-26); Whitaker, 1980: 39; Hanlon et al., 1983: 638 (fig. 4); Roper et al., 1984: 98; Haimovici \& Perez, 1991: 227; Costa \& Fernandes, 1993: 753 (tab. 1); DiMarco \& Hanlon, 1997: 93 (fig. 2); Anderson, 2000: 606 (tab. 2); Jackson \& Forsythe, 2002: 677; Perez et al., 2002: 271 (tab. 2); Pineda et al., 2002: 71 (tab. 1); Cardoso et al., 2005: 373 (tab. 4); Martins \& Perez, 2006: 24 (fig. 1); Perez et al., 2006: 750 (fig. 1); Rodrigues \& Gasalla, 2008: 634 (tab. 1).

Doryteuthis plei [sic]: Brakoniecki, 1984: 436; Haimovici \& Andriguetto, 1986_ENREF_13: 5 (fig. 1); Grassi et al., 1990: 260; Vecchione et al., 2005: 25 (tab. 2); Jereb \& Roper, 2010: 55 (fig. 83); Marian, 2010_ENREF_20: 2 (fig. 1); de Pasquier \& Carroz, 2012: 37 (fig. 4); de Aguiar et al., 2012: 17 (tab. 1); Marian, 2012: 250 (figs. 1-21); Marian \& Domaneschi, 2012: 93 (figs. 1-20); de Luna Sales et al., 2013: 294 (fig. 1); Barcellos \& Gasalla, 2014: 3 (figs. 1, 2, 3 and 6).

Doryteuthis pleii: Allcock et al., 2015: 18 (tab. 2); Willems et al., 2015: 28 (tab. 2).

Type locality: Martinique, West Indies, western North Atlantic Ocean.

Holotype: Muséum National d'Histoire Naturelle, Paris [despite the label of the specimen thought to be the holotype is missing (Cohen, 1976)].

Material examined: Brazil; Rio de Janeiro: off São João da Barra, MZSP 15294, 24 spm. ("Exp. Dep. Zool." coll., July 1963). off Farol de São Thomé, $22^{\circ} 08^{\prime} S$, 4100'W, MZSP 27095, 22 m, 1 spm. (R/V "W. Besnard" coll., 12 March 1971). off Cabo Frio, 22 $51^{\prime} \mathrm{S}, 41^{\circ} 52^{\prime} \mathrm{W}$, MZSP 27092, 48 m, 17 spm. (R/V "W. Besnard" coll.,

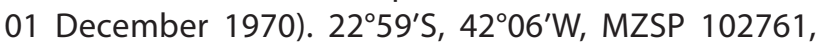
25-33 m, 2 spm. (1 June 1987). off Saquarema, $23^{\circ} 02^{\prime} S$, 42³7'W, MZSP 101893, 50-62 m, 1 spm. (19 November 2007). off Niterói, $23^{\circ} 05^{\prime} S, 43^{\circ} 06^{\prime} W$, MZSP 110029, 30-50 m, 2 spm. (Mendonça Jr. coll., 05 December 2012). off Angra dos Reis, Vila Velha Beach, MZSP 27063, 1 spm. ("Exp. Dep. Zool." coll., 21 July 1966). off Paraty, 2311'S,

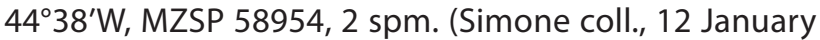
2006). MZSP 27061, 13 spm. (July 1983). MZSP 32965, 2 spm. (Fishermen coll., March 2001). São Paulo: off Ubatuba, MZSP 27076, 8 spm. (Boffi coll., 08 February 1970). MZSP 101892, 1 spm. (Caires coll., 2011). MZSP 27084, 2 spm. (Local fishermen coll., 12 March 1970). Lázaro Beach, MZSP 27087, 2 spm. (Sazima coll., 04 April

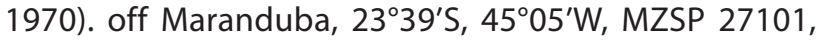
28-32 m, 4 spm. (R/V "W. Besnard" coll., 05 December 1970). off Santos, MZSP 90936, 2 spm. (Cunha coll., March 2009). MZSP 27097, 42 m, 4 spm. (R/V “W. Besnard” coll., 05 December 1970). MZSP 86427, 8 spm. (R/V “Miragem Boat" coll., March 1988). off Praia Grande, Boqueirão,

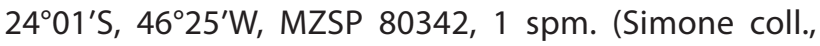
1997). $24^{\circ} 05^{\prime} S, 46^{\circ} 20^{\prime} \mathrm{W}, \mathrm{MZSP} 110145,2 \mathrm{spm}$. (10 April 2012). off São Sebastião, Alcatrazes archipelago. $24^{\circ} 07^{\prime} \mathrm{S}, 45^{\circ} 40^{\prime} \mathrm{W}, \mathrm{MZSP} 102400,46 \mathrm{~m}, 3 \mathrm{spm}$. (Amaral

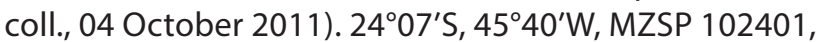
46 m, 2 spm. (Amaral coll., 04 October 2011). 24²4'S, $46^{\circ} 05^{\prime} \mathrm{W}, \mathrm{MZSP} 27159,52 \mathrm{~m}, 25 \mathrm{spm}$. (R/V "W. Besnard" 


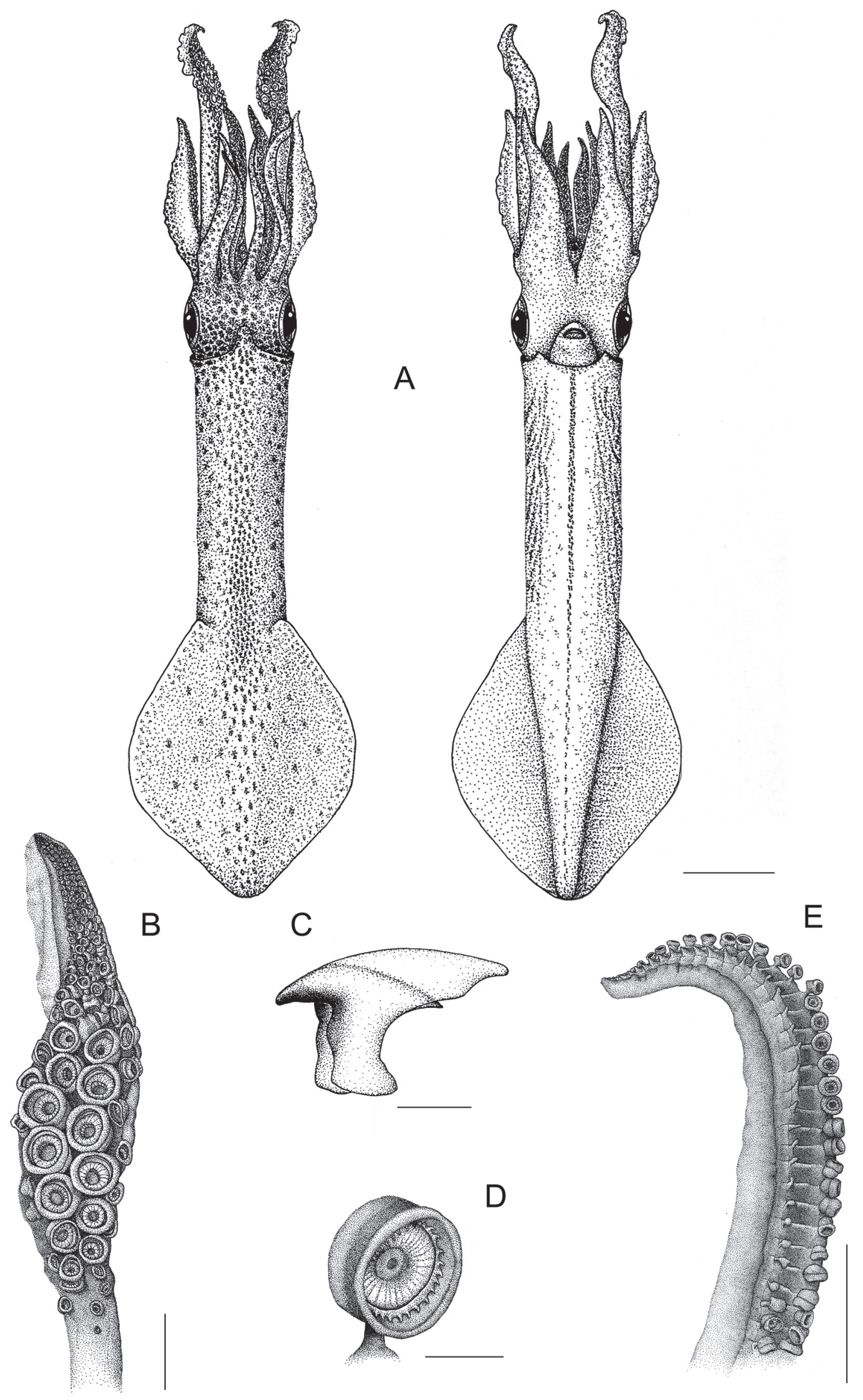

Figure 1. Doryteuthis pleii general features. (A) Dorsal (left) and ventral (right) whole view of a male, scale $=20 \mathrm{~mm}$; (B) Tentacular club, scale $=5 \mathrm{~mm}$; (C) Upper beak, scale = $2 \mathrm{~mm}$; (D) Sucker from tentacular club, scale = $1 \mathrm{~mm}$; (E) Left ventral arm showing hectocotylized portion, scale $=5 \mathrm{~mm}$. 


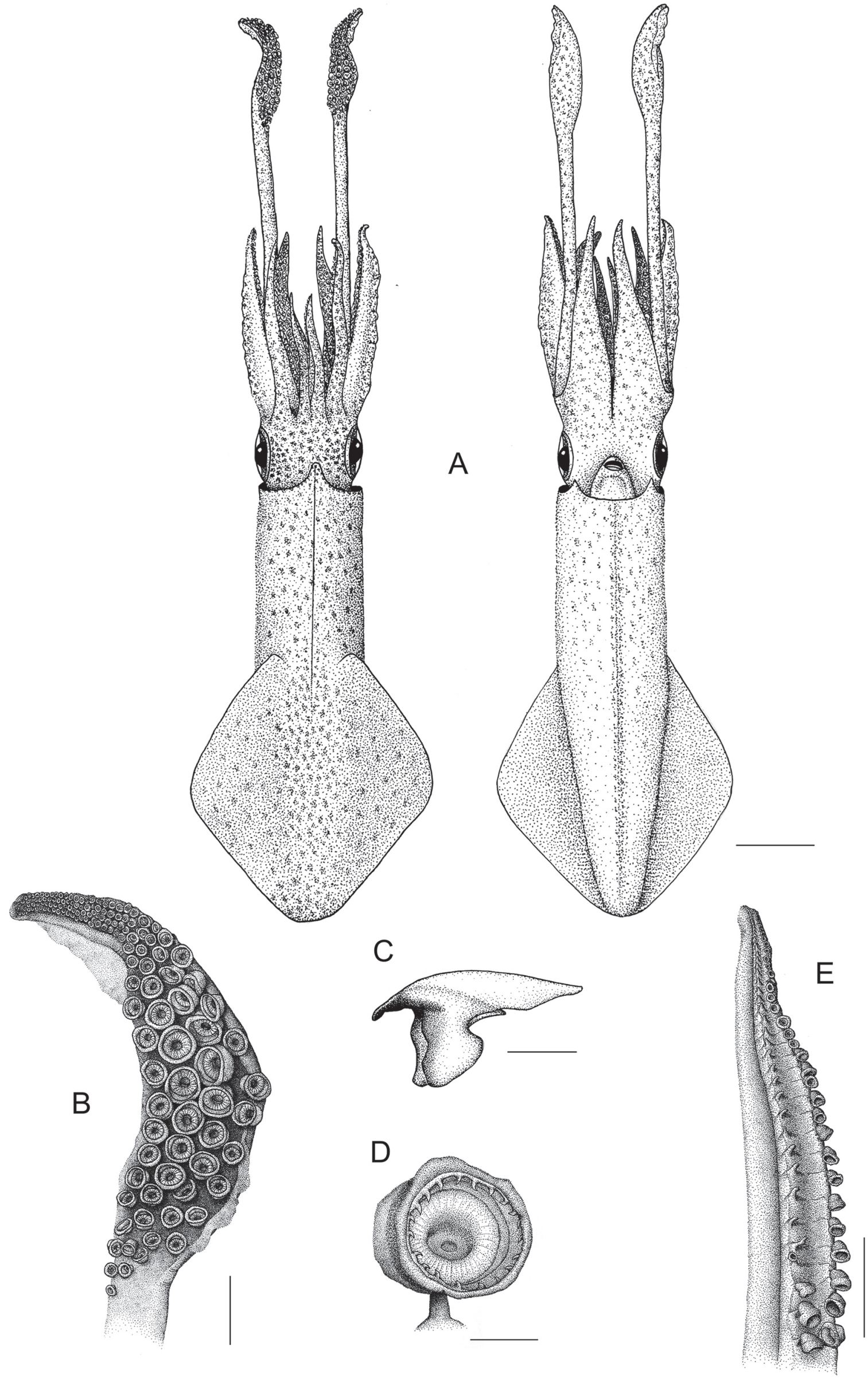

Figure 2. Doryteuthis sanpaulensis general features. (A) Dorsal (left) and ventral (right) whole view of a male, scale $=20 \mathrm{~mm}$; (B) Tentacular club, scale $=5 \mathrm{~mm}$; (C) Upper beak, scale = $2 \mathrm{~mm}$; (D) Sucker from tentacular club, scale = $1 \mathrm{~mm}$; (E) Left ventral arm showing hectocotylized portion, $\mathrm{scale}=5 \mathrm{~mm}$. 
coll., 21 January 1971). off Itanhaém, $24^{\circ} 24^{\prime} S, 46^{\circ} 18^{\prime} \mathrm{W}$, MZSP 27093, 45 m, 13 spm. (R/V "W. Besnard" coll., 05 March 1971). off Peruíbe, $24^{\circ} 20^{\prime} S, 46^{\circ} 49^{\prime} W$, MZSP 27094, 79 m, 28 spm. (R/V “W. Besnard" coll., 06 March 1971). $24^{\circ} 38^{\prime} \mathrm{S}, 45^{\circ} 05^{\prime} \mathrm{W}, \mathrm{MZSP} 27102,102 \mathrm{~m}, 6 \mathrm{spm}$. (R/V "W. Besnard" coll., 06 March 1971). off Barra do Una, 244ㅇ's, 4649'W, MZSP 27096, 38 m, 10 spm. (R/V "W. Besnard" coll., 06 December 1970). off Cananéia, Castilho Island - ESEC Tupiniquins, $25^{\circ} 00^{\prime} \mathrm{S}$, $47^{\circ} 55^{\prime} \mathrm{W}$, MZSP 83165, 5 spm.(Guimarães, Lima \& Amaral coll.,

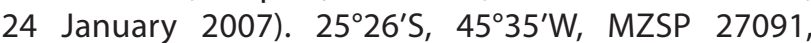
125-128 m, 10 spm. (R/V "W. Besnard" coll., 05 July

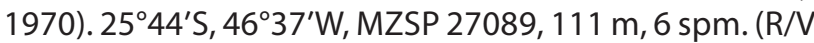
"W. Besnard" coll., 04 March 1971). Paraná: off Pontal

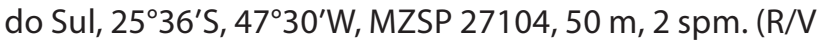

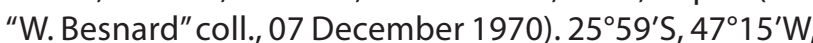
MZSP 27105, 77-82 m, 4 spm. (R/V "W. Besnard" coll., 07 December 1970). Santa Catarina: São Francisco

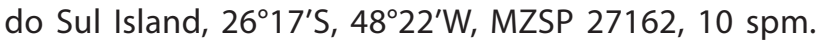
(R/V “W. Besnard" coll., February 1977). off Itajaí, MZSP 92949, 51 m, 2 spm. (R/V "NPq Soloncy Moura” coll., 09 November 2009). off Imbituba, $2^{\circ} 18^{\prime} S$, $48^{\circ} 34^{\prime} \mathrm{W}$, MZSP 27090, 50 m, 12 spm. (R/V “W. Besnard" coll., 01 March 1971). Rio Grande do Sul: off Balneário Pinhal, 30³7'S, $49^{\circ} 25^{\prime} \mathrm{W}$, MZSP 27058, $150 \mathrm{~m}, 4$ spm. (GEDIP Project coll., 05 August 1972).

Distribution: Cape Hatteras $\left(36^{\circ} \mathrm{N}\right)$ to northern Argentina $\left(35^{\circ} \mathrm{S}\right)$ usually associated with Brazilian warm current. Also including Gulf of Mexico, Caribbean Sea, Bermuda and Bahamian and Caribbean islands (Jereb \& Roper, 2010).

\section{Description}

Mantle: Cylindrical; muscular ( $91 \mathrm{~mm}$; Table 1); usually long and slender (Fig. 1A), 4-times longer than wide.

Head: Eye diameter approximately $13 \%$ of mantle length (Table 2). Width similar to length values.

Chromatophores: Widely distributed, with higher densities on dorsal region including head (around eyes), dorsal midline of mantle (highly concentrated beginning of fins), fins and arms/tentacles. Mature males presenting striped pattern along ventral surface of mantle (Fig. 1A).

Fins: Rhomboid, slightly ovate comprising 48\% ML (Table 2), connected posteriorly to posterior end of mantle (Fig. 1A).

Arms: Muscular, broad at base and gradually tapering into thin tips; formula III $>$ IV $>$ II $>$ I ( lengths: $33 \mathrm{~mm}$, $30 \mathrm{~mm}, 29 \mathrm{~mm}, 23 \mathrm{~mm}$ ); biserial suckers. Hectocotylized portion $\sim 39 \%$ of left ventral arm length reaching arm's tip (Table 2), only left row of suckers modified into small peduncles. Bar between row of peduncles and row of suckers connecting them in ladder pattern (Fig. 1E).
Tentacle: Club long and thin, 28\% ML (Table 2), carpus, manus and dactylus easily defined; 4 rows of suckers diagonally distributed along club, more visible at manus and dactylus regions; 2 central rows at manus region much bigger than other suckers. Each sucker with small chitin ring of teeth inside. Longitudinal muscle around sucker visible (Figs. 1B, 1D).

Circulatory system: Normal Loliginidae form (Fig. 6A). One central systemic croissant-shaped heart; two smaller peripheral branchial round hearts associated to gills. Two aortae: (i) anterior aorta delivering blood to head, arms and tentacles; (ii) posterior aortic trunk, divided in: (a) visceral aorta, sending blood to posterior part of mantle and visceral organs; (b) mantle aorta, distributing blood to anterior region of mantle. Blood return to heart (i) from posterior vena cava and (ii) from lateral nephridial vessel, both ending in a branchial heart. Branchial artery leaving branchial heart, and ending in gills complex; after this, returning to heart through branchial vein (Fig. 3A).

Digestive system (Figs. 5, 6): Pigmented tip of upper beak short and robust (Fig. 1C). Radula usual for the family; rachidian and first lateral teeth bearing primary large projection and secondary smaller one(s) (Fig. 4A). Usual digestive system for Loliginidae: buccal bulb occupying half of inner head volume. Esophagus narrow, running straight posteriorly along $\sim 1 / 5$ of mantle length. Stomach spherical, wall weakly muscular, occupying $\sim 20 \%$ of visceral volume, located in middle level of visceral sac; posterior caecum wide, extending up to posterior mantle space. Intestine narrow, originating in $Y Y$ region of stomach, performing simple loop in anterior region of visceral sac. Anus with two papillae, located in middle level of pallial cavity, preceded by short flexible stalk. Digestive gland wide and located between four retractile muscles of head and funnel. Esophagus passing through digestive gland ventro-dorsally.

Male genital system: Normal genital system for Loliginidae, including characters of penis, Needham sac, spermatophoric gland and a single testicle (Figs. 6A, 6B). Hectocotylus described above.

Female genital system: Normal genital system for Loliginidae, including characters of vagina, oviduct, oviducal gland, ovary, a pair of nidamental glands and pair of accessory nidamental glands (Fig. 5). Nidamental glands more uniform, with no slender tip (Fig. 5B).

Central nervous system (Fig. 3B): Central mass slightly divided into anterior and posterior regions. Giant fibers running from posterior end, along dorsal portion of mantle; stellate ganglion visible beneath gills. One pair of optic lobes mushroom-shaped, flanking central mass on each side. Several nerves running anteriorly towards arms and tentacles, forming ring around buccal bulb (Fig. 3B: ib). 
Table 1. Biometric measurements of Doryteuthis pleii. ML: mantle length (mm); FL: fins length (mm); ED: eye diameter (mm); Al, All, Alll, AIV: arms length dorsally to ventrally $(\mathrm{mm})$; HtL: hectocotylus length $(\mathrm{mm})$; $\mathrm{CL}$ : tentacular club length $(\mathrm{mm})$.

\begin{tabular}{|c|c|c|c|c|c|c|c|c|c|c|c|c|c|c|c|c|c|c|c|c|c|}
\hline \multirow{2}{*}{\multicolumn{2}{|c|}{$\begin{array}{c}\text { Individual } \\
\text { Sex }\end{array}$}} & 1 & 2 & 3 & 4 & 5 & 6 & 7 & 8 & 9 & 10 & 11 & 12 & 13 & 14 & 15 & 16 & 17 & 18 & 19 & 20 \\
\hline & & $0^{*}$ & q & 운 & q & 운 & $0^{\pi}$ & $0^{*}$ & $0^{\pi}$ & q & $0^{\pi}$ & $0^{*}$ & $0^{\pi}$ & $0^{x}$ & $0^{*}$ & 운 19 & q & 운 & q & & $0^{*}$ \\
\hline \multicolumn{2}{|c|}{ MI } & 165 & 124 & 122 & 147 & 92 & 87 & 88 & 39 & 72 & 80 & 61 & 49 & 55 & 50 & 53 & 112 & 79 & 101 & 82 & 111 \\
\hline & 87 & 64,6 & 60,2 & 78,1 & 53,8 & 51,4 & & 20,4 & & & 31,9 & 26,1 & 27,2 & 24,3 & 25,7 & & 32,3 & 4,7 & 2,5 & 51,5 \\
\hline \multicolumn{2}{|l|}{ ED } & 14,6 & 13,8 & 14 & 15,3 & 11,1 & 10,3 & 10,3 & 7,5 & 8,7 & 10,3 & 10 & 7,3 & 8 & 7,6 & 9,4 & 12,6 & 9 & 12,2 & 9,4 & 11 \\
\hline \multirow[t]{6}{*}{ LEFT } & Al & 34,6 & 32,6 & 31,4 & 35,1 & 25,3 & 26,4 & 26,5 & 14 & 22,6 & 22,6 & 21,9 & 14,2 & 16 & 18,7 & 18,8 & 26,5 & 19,1 & 5,4 & 20,6 & 30,7 \\
\hline & All & 40,5 & 36,2 & 37,1 & 40,8 & 34,5 & 32,9 & 32,4 & 16,2 & 27,8 & 26,9 & 23,9 & 17,1 & 23,8 & 10,5 & 21,4 & 31,3 & 20,3 & 30,4 & 23 & 36,6 \\
\hline & AllI & 43,4 & 38,7 & 37,3 & 45,5 & 39,1 & 36 & 36,4 & 20,9 & 32,4 & 37,1 & 27,7 & 22,9 & 26,5 & 24,9 & 26 & 35,5 & 7,3 & 4,8 & 30,1 & 39,8 \\
\hline & AIV & 40,4 & 36,6 & 30,9 & 42,1 & 32,7 & 33,9 & 33,2 & 19,1 & 28,6 & 33,1 & 27 & 20,8 & 21,4 & 23,7 & 23 & 33 & 2,1 & 32,8 & 25,2 & 37,1 \\
\hline & HtL & 15,8 & - & - & - & - & 10,8 & 13 & 5,2 & - & 13,9 & 9,4 & 9,5 & 8 & 9,4 & - & - & - & - & - & 18,7 \\
\hline & $\mathrm{CL}$ & 25,2 & 27,3 & 26,3 & 32 & 34,5 & 24,9 & 22,8 & 12,8 & 20,4 & 24,8 & 21,6 & 16,5 & & 15,7 & 24 & 28,2 & 18,2 & 28,8 & 23,8 & 25,4 \\
\hline \multirow[t]{5}{*}{ RIGHT } & I Al & 34,6 & 31,1 & 30,4 & 28,7 & 28,5 & 27 & 24,2 & 13,4 & 21,1 & 28,4 & 20,1 & 14,5 & 16,3 & 18,6 & 17 & 27 & & 26,4 & 20,6 & 31,5 \\
\hline & All & 39,6 & 37,4 & 34,6 & 41 & 33,9 & 34,2 & 29,6 & 16,5 & 26,6 & 31,1 & 27,2 & 21,4 & 20,8 & 21,7 & 13,2 & 32,9 & 25,4 & 28,6 & 25,3 & 39,3 \\
\hline & AlII & 42,9 & 38,2 & 38,1 & 43 & 41,2 & 38,7 & 33,5 & 22,3 & 33,1 & 37,5 & 28 & 23,4 & & 2 & 5,1 & 38,5 & 7,6 & 3,7 & 9,5 & 41,3 \\
\hline & AIV & 36,9 & 37,4 & 31,8 & 40,1 & 37,8 & 32 & 34,5 & 19,3 & 31 & 34,1 & 28,9 & 22,3 & 15,9 & 24 & 23,8 & 34,8 & & 34,2 & 26,5 & 34 \\
\hline & $\mathrm{CL}$ & 24,6 & 27,9 & 25,9 & 32,8 & 35,3 & 25,5 & 23,5 & 13,3 & 22,3 & 25,3 & 22,1 & 15,8 & 17,5 & 15,4 & 24,9 & 27 & 18,4 & 28,7 & 21,1 & 24,3 \\
\hline \multicolumn{2}{|c|}{ Individual } & 21 & 22 & 23 & 24 & 25 & 26 & 27 & 28 & 29 & 30 & 31 & 32 & 33 & 34 & 35 & 36 & 37 & 38 & 39 & 40 \\
\hline \multicolumn{2}{|c|}{ Sex } & $0^{7}$ & $0^{n}$ & 앙 & $0^{\pi}$ & 웅 & 웅 & 운 & 웅 & 웅 & $0^{\pi}$ & 웅 & 웅 & q & 우 & 웅 & q & q & 웅 & 웅 & q \\
\hline & 117 & 90 & 136 & 104 & 84,4 & 67,2 & 65 & 66,3 & 60,1 & 62,8 & 72 & 74,5 & 151 & 175 & 121 & 80,1 & 70,4 & 7,4 & 102 & 77 \\
\hline & 47,7 & 40,1 & 72,8 & 54,4 & 33 & 28,9 & 23,7 & 26,6 & 25,3 & 26,3 & 40,9 & 40 & 7 & 90 & 62 & 3 & 3 & 36,4 & 46 & 30,5 \\
\hline \multicolumn{2}{|l|}{ ED } & 10,9 & 10,9 & 12,6 & 10,5 & 12,9 & 10,7 & 11,4 & 10,9 & 10,9 & 11,9 & 11,3 & 10,6 & 15,5 & 15,7 & 13,5 & 12,2 & 11 & 11,5 & 13 & 10 \\
\hline \multirow[t]{6}{*}{ LEFT } & Al & 29,1 & 25,6 & 31,1 & - & 21,2 & 19,7 & 18,6 & 19,9 & 19,5 & 18,3 & 18,1 & 21 & - & - & 31,7 & 24,2 & 19,2 & 21,7 & 22,8 & 16,3 \\
\hline & All & 34,4 & 31,9 & 28,5 & 34,6 & 29,5 & 28 & 21,9 & 26,4 & 24,4 & 23,5 & 29 & 31,3 & - & - & 39 & 29,9 & 26 & 27,5 & 28,4 & 19,9 \\
\hline & AllI & 39,7 & 36,1 & 43,6 & 38,5 & 33 & 27,4 & 27,2 & 26,8 & 25,8 & 25 & 35,3 & 33 & - & - & 43 & 31,9 & 27,2 & 29,5 & 37,6 & 25 \\
\hline & AIV & 36,4 & 30,6 & 34,8 & 37,7 & 34,2 & 24,9 & 30,1 & 24,8 & 26,3 & 26 & 35,4 & 34 & - & - & 42,8 & 30,4 & 26,3 & 29 & 32 & 26 \\
\hline & HtL & 15,9 & 11,8 & - & 16,3 & - & - & - & - & - & 8,2 & - & - & - & - & - & - & - & - & - & - \\
\hline & CL & 25,5 & 22,9 & 31,5 & 24,3 & 20,3 & 21,6 & 22,4 & 23,4 & 19,8 & 20,1 & 19,6 & 22,2 & 33,4 & 35,2 & 30,7 & 25,4 & 18,8 & 23,1 & 25,5 & 19,5 \\
\hline \multirow[t]{5}{*}{ RIGHT } & I Al & 30,3 & 26,9 & 32 & 30,9 & 22 & 20,6 & 18,6 & 19,9 & 19,6 & 19,4 & 20,5 & 21 & - & - & 32 & 24,2 & 20 & 22 & 25,6 & 16 \\
\hline & All & 34,6 & 33,5 & 25,4 & 37,3 & 30,8 & 26,8 & 22,7 & 24,6 & 25,8 & 26,2 & 28,7 & 30 & - & - & 39,7 & 27 & 27 & 26 & 25,7 & 20 \\
\hline & AllI & 30,9 & 35,2 & 42,3 & 40,3 & 33 & 26,9 & 29,4 & 29,7 & 25,8 & 28 & 33 & 35 & - & - & 45,5 & 32 & 27,3 & 31 & 35 & 25,3 \\
\hline & AIV & 37,2 & 35,1 & 40,4 & 39,2 & 33 & 21,8 & 27,9 & 27,4 & 26,8 & 24 & 33,2 & 34 & - & - & 42 & - & 27,1 & 30 & 31,7 & 25,8 \\
\hline & $\mathrm{CL}$ & 25,1 & 22,6 & 31,2 & 24,2 & 20,1 & 21,5 & 22,3 & 23,5 & 19,9 & 20 & 19,8 & 22,3 & 33,4 & 35,2 & 30,5 & 25,6 & 18,7 & 23,3 & 25,3 & 19,6 \\
\hline
\end{tabular}

Table 2. Proportions between characters of Doryteuthis pleii. FL-ML: Proportion between fin length and mantle length (\%); ED-ML: Proportion between eye diameter and mantle length (\%); HtL-Lft AIV: Proportion between hectocotylized portion and left ventral arm length (\%). CL-ML: Proportion between tentacular club length and mantle length (\%).

\begin{tabular}{|c|c|c|c|c|c|c|c|c|c|c|c|c|c|c|c|c|c|c|c|c|}
\hline & 1 & 2 & 3 & 4 & 5 & 6 & 7 & 8 & 9 & 10 & 11 & 12 & 13 & 14 & 15 & 16 & 17 & $\begin{array}{l}18 \\
0\end{array}$ & 19 & $\begin{array}{l}20 \\
\sigma^{7}\end{array}$ \\
\hline FL-ML & 527 & 521 & 493 & 531 & 585 & 501 & 5 & 523 & 567 & 58 & 523 & 533 & 495 & 486 & 485 & 487 & 40.9 & 4 & 396 & 46,4 \\
\hline ED-ML & 8,85 & 11,1 & 11,5 & 10,4 & 12,1 & 11,8 & 11,7 & 19,2 & 12,1 & 12,9 & 16,4 & 14,9 & 14,5 & 15,2 & 17,7 & 11,3 & 11,4 & 12,1 & 11,5 & 9,91 \\
\hline HtL-Lft AIV & 39,1 & - & - & - & - & 31,9 & 39,2 & 27,2 & - & 42 & 34,8 & 45,7 & 40,2 & 39,7 & - & - & - & - & - & 50,4 \\
\hline \multirow[t]{3}{*}{ CL-ML } & 15,1 & 22,3 & 21,4 & 22 & 37,9 & 29 & 26,3 & 33,5 & 29,7 & 31,3 & 35,8 & 33 & 32,7 & 31,1 & 46,1 & 24,6 & 23,2 & 28,5 & 27,4 & 22,4 \\
\hline & 21 & 22 & 23 & 24 & 25 & 26 & 27 & 28 & 29 & 30 & 31 & 32 & 33 & 34 & 35 & 36 & 37 & 38 & 39 & 40 \\
\hline & $0^{\pi}$ & $0^{n}$ & q & $0^{n}$ & $q$ & q & q & q & q & $0^{n}$ & $q$ & q & $q$ & q & q & q & q & q & q & q \\
\hline FL-ML & 40,8 & 44,6 & 53,5 & 52,3 & 39,1 & 43 & 36,5 & 40,1 & 42,1 & 41,9 & 56,8 & 53,7 & 49,8 & 51,4 & 51,4 & 43,7 & 45,5 & 41,6 & 45,1 & 39,6 \\
\hline ED-ML & 9,32 & 12,1 & 9,26 & 10,1 & 15,3 & 15,9 & 17,5 & 16,4 & 18,1 & 18,9 & 15,7 & 14,2 & 10,3 & 8,97 & 11,2 & 15,2 & 15,6 & 13,2 & 12,7 & 13 \\
\hline HtL-Lft AIV & 43,7 & 38,6 & - & 43,2 & - & - & - & - & - & 31,5 & - & - & - & - & - & - & - & - & - & - \\
\hline CL-ML & 21,6 & 25,3 & 23,1 & 23,3 & 23,9 & 32,1 & 34,4 & 35,4 & 33 & 31,9 & 27,4 & 29,9 & 22,2 & 20,1 & 25,4 & 31,8 & 26,6 & 26,5 & 24,9 & 25,4 \\
\hline
\end{tabular}

\section{Doryteuthis sanpaulensis (Brakoniecki, 1984) (Figs. 2, 3, 5, 6)}

\section{Synonymy see Brakoniecki (1984). Complement:}

Loligo sanpaulensis Brakoniecki, 1984: 441-446 (figs. 3-4); Roper et al., 1984: 102; Haimovici \& Perez, 1991: 227; Costa \& Fernandes, 1993: 753 (tab. 1);
Andriguetto \& Haimovici, 1996: 444; Pineda et al., 1996: 88 (fig. 5); Sanchez et al., 1996: 540 (fig. 17); Anderson, 2000: 606 (tab. 2); Barón \& Ré, 2002: 270 (figs. 1 and 5); Herke \& Foltz, 2002: 106 (tab. 1); Pineda et al., 2002: 71 (tab. 1); Barón, 2003a: 222 (fig. 1); Barón, 2003b: 1350 (figs. 4, 6 and 8); Rodrigues \& Gasalla, 2008: 634 (tab. 2); Vidal et al., 2010: 1348 (tab. 1). 
Doryteuthis sanpaulensis: Vecchione et al., 2005: 25 (tab. 2); Jereb \& Roper, 2010: 69 (fig. 97); de Aguiar et al., 2012: 17 (tab. 1); de Luna Sales et al., 2013: 294 (fig. 1); Crespi-Abril et al., 2014: 15 (tab. 1).

Type locality: San Matias Gulf, Argentina, $41^{\circ} 47^{\prime} \mathrm{S}$, $63^{\circ} 35^{\prime} \mathrm{W}$.
Holotype: USNM 815464.

Material examined: Brazil; Rio de Janeiro: off Farol de São Thomé, MZSP 101894, 1 spm. (Nunan coll., 26 January 2007). off Maricás Island, $23^{\circ} 01^{\prime} S, 42^{\circ} 50^{\prime} \mathrm{W}$, MZSP 101891 ,

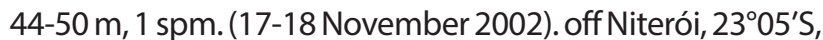
43은 $\mathrm{W}$, MZSP 110019, 30-50 m, 1 spm. (Mendonça Jr
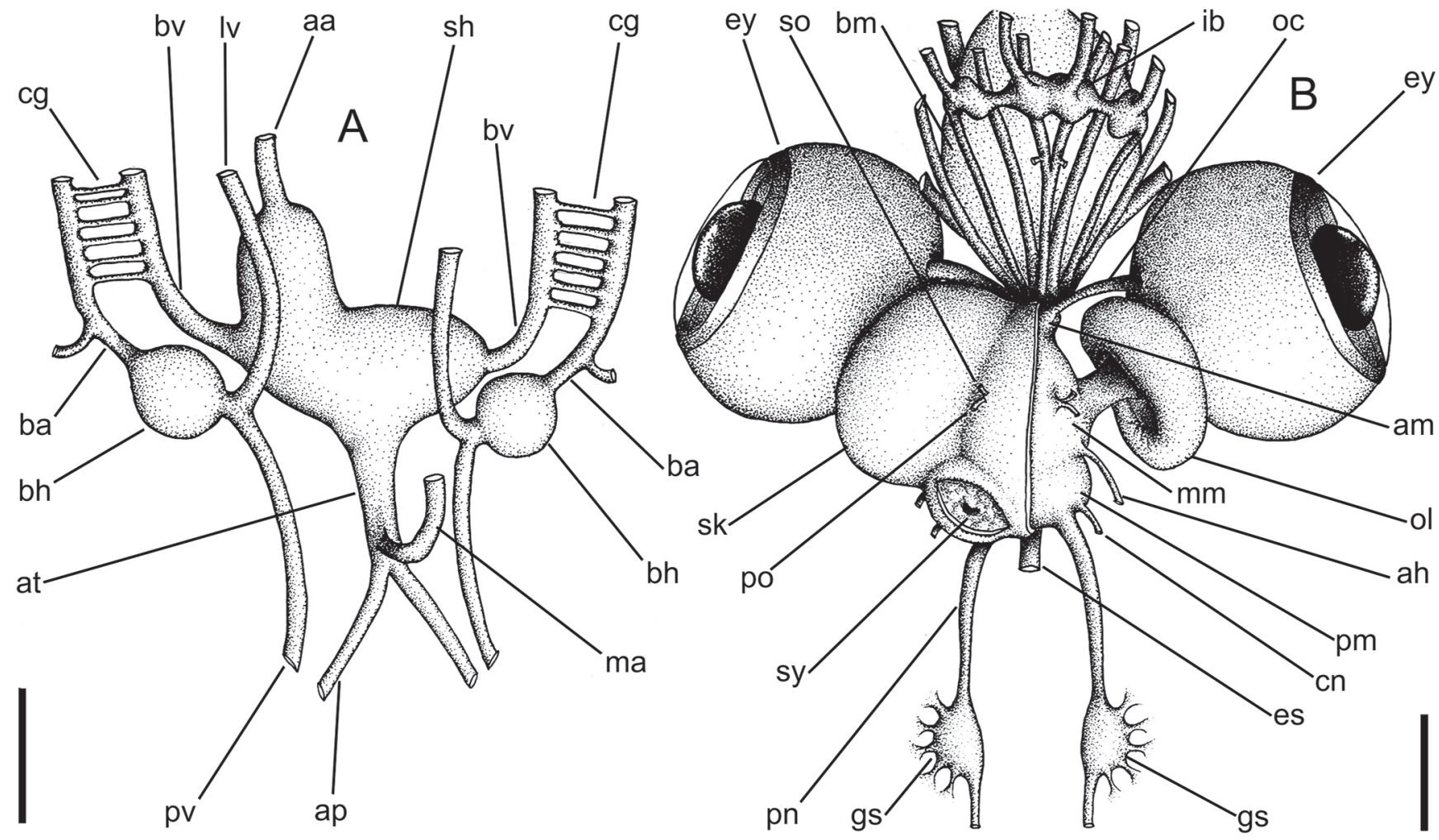

Figure 3. (A) Central vascular system representing both species, ventral view; (B) Central nervous system representing both species, ventral view. Scales: $=5 \mathrm{~mm}$.
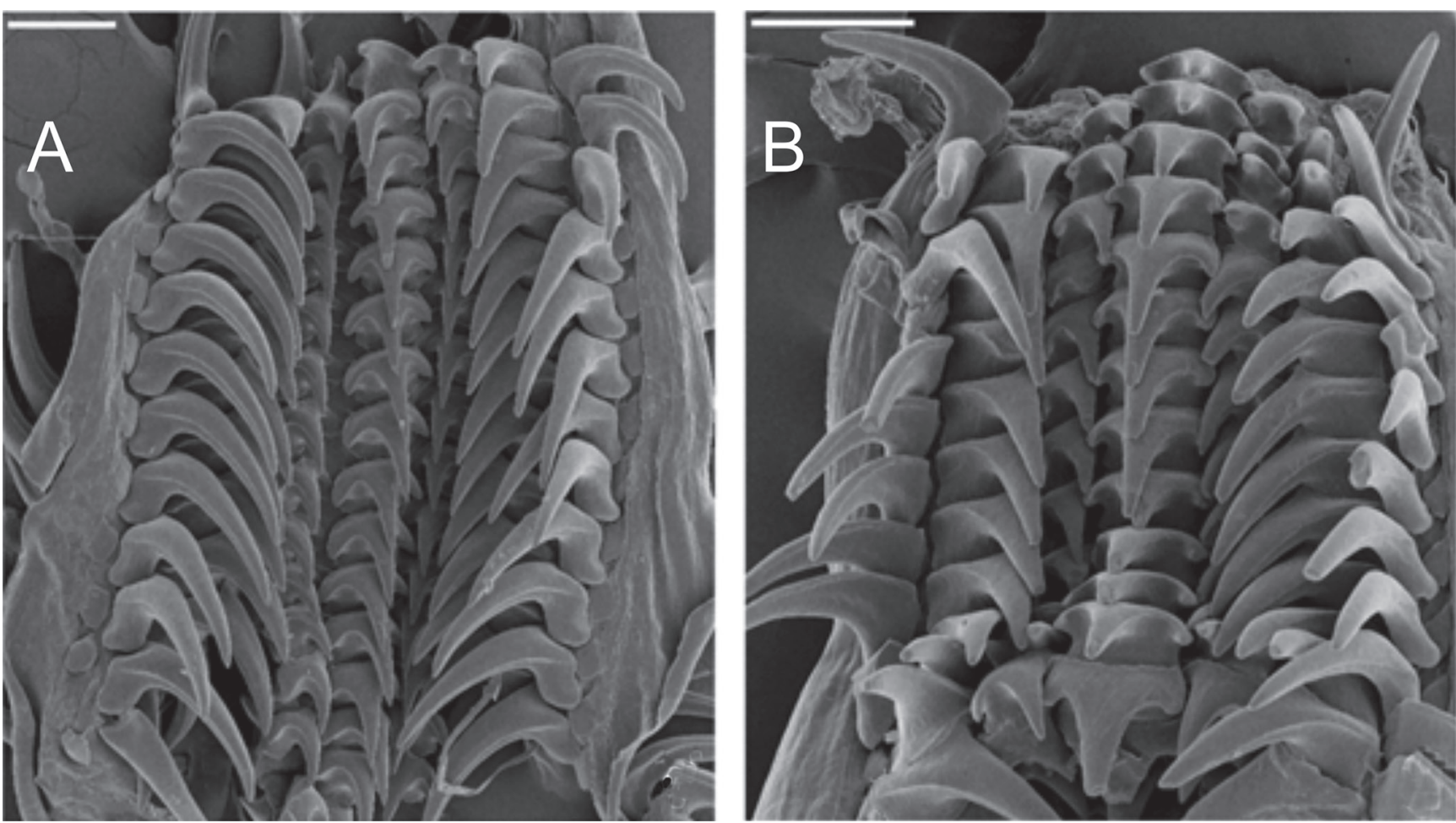

Figure 4. Radulae in SEM: (A) Doryteuthis pleii; (B) Doryteuthis sanpaulensis. Scales: $=200 \mu \mathrm{m}$. 


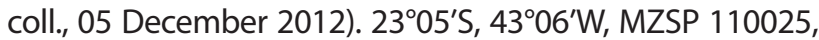
30-50 m, 20 spm. (Mendonça Jr coll., 05 December 2012). off Paraty, MZSP 32966, 2 spm. (Fishermen coll., March 2001). São Paulo: off Ubatuba, Itaguá Beach, MZSP 26409, 2 spm. (Figueiredo coll.). MZSP 27083, 19 spm. (Monfouchet coll., 12 March 1970). off São Sebastião, Alcatrazes Island, MZSP

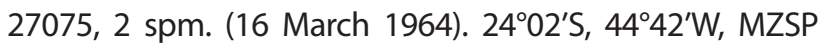
27160, 80-90 m, 25 spm. (R/V “W. Besnard” coll., 11 August 1970). MZSP 47280, 2 spm. off Santos, MZSP 34506, 10-15 m, 6 spm. (Cunha coll., May 1998). MZSP 34645, 130-150 m, 1 spm. (Cunha coll., February 2002). MZSP 47289, 18 m, 13 spm. (R/V “Emilia" coll., November 1976). MZSP 91389, 3 spm. (Cunha coll., June 2009). MZSP 107571, 20 m, 30 spm. (Cunha coll., June 2009). off Boqueirão, $24^{\circ} 01^{\prime} S, 46^{\circ} 25^{\prime} \mathrm{W}$, MZSP 80343, 11 spm. (Simone coll., 1997). MZSP 27321, 6 m, 3 spm. (Simone coll., 10 January 1990). off Iguape, $25^{\circ} 10^{\prime} \mathrm{S}, 46^{\circ} 45^{\prime} \mathrm{W}, \mathrm{MZSP} 18639,7 \mathrm{spm}$. (R/V "W. Besnard" coll., 30 May 1970). Santa Catarina: off Itajaí, MZSP 92950, 51 m, 11 spm. (R/V "NPq Soloncy Moura" coll., 09 November 2009). off Florianópolis, $27^{\circ} 09^{\prime} \mathrm{S}, 47^{\circ} 41^{\prime} \mathrm{W}$, MZSP 27135, 115 m, 2 spm. (R/V "W. Besnard" coll., 16 August 1970). $27^{\circ} 56^{\prime} \mathrm{S}, 48^{\circ} 29^{\prime} \mathrm{W}, \mathrm{MZSP} 18680,8 \mathrm{spm}$. (R/V "W. Besnard" coll., 17 December 1970). off Laguna, $28^{\circ} 32^{\prime} \mathrm{S}, 48^{\circ} 11^{\prime} \mathrm{W}$, MZSP 18638, 4 spm. (R/V “W. Besnard” coll., 03 June 1970). off Balneário Gaivota, 2913'S, 49³5'W, MZSP 27133, 20 m, 2 spm. (R/V "W. Besnard" coll., 31 January 1972). 29¹3'S, 49³5'W, MZSP 27148, 19 m, 10 spm. (R/ “'W. Besnard" coll., 06 April 1972). $29^{\circ} 16^{\prime} \mathrm{S}, 49^{\circ} 14^{\prime} \mathrm{W}, \mathrm{MZSP} 27143,51 \mathrm{~m}, 3$ spm. (R/V “W. Besnard” coll., 30 January 1972). 29²3'S, 49¹6'W, MZSP 27137, 50 m, 4 spm. (R/V “W. Besnard" coll., 06 April 1972). Rio Grande do Sul: off Arroio do Sal, 2952'S, 4841'W, MZSP 18603, 4 spm. (R/V "W. Besnard" coll., 05 March 1969). off Capão da Canoa, $29^{\circ} 58^{\prime} S, 49^{\circ} 18^{\prime} W$, MZSP 27144, 92 m, 5 spm. (R/V“W. Besnard”coll., 29 January 1972). off Tramandaí, MZSP 26410, 3 spm. (Phonlor coll., 1974). off Cidreira, MZSP 26411, 10 m, 1 spm. (Phonlor coll., July 1974). MZSP 26408, 10 m, 1 spm. (Phonlor coll., 08 October 1974). off Balneário Pinhal, $30^{\circ} 16^{\prime} \mathrm{S}, 50^{\circ} 09^{\prime} \mathrm{W}, \mathrm{MZSP} 27138$, 22 m, 2 spm. (R/V "W. Besnard" coll., 28 January 1972). $30^{\circ} 22^{\prime} \mathrm{S}, 49^{\circ} 36^{\prime} \mathrm{W}, \mathrm{MZSP} 18589,18 \mathrm{spm}$. (R/V "W. Besnard" coll., 05 December 1968). 30²7'S, 49²8'W, MZSP 27130, 71 m, 6 spm. (R/V "W. Besnard" coll., 28 January 1972). off Solidão, 3047'S, 50²8'W, MZSP 27145, 17 m, 8 spm. (R/V "W. Besnard" coll., 26 January 1972). 3053'S, 50¹6'W, MZSP 27132, 60 m, 2 spm. (R/V "W. Besnard" coll., 26 January 1972). off Mostardas, $31^{\circ} 09^{\prime} \mathrm{S}, 50^{\circ} 43^{\prime} \mathrm{W}$, MZSP 27131, $20 \mathrm{~m}$, 12 spm. (R/V "W. Besnard" coll., 07 August 1972). 31¹2'S, 50³5'W, MZSP 27141, 59 m, 5 spm. (R/V "W. Besnard" coll., 29 January 1972). off Tavares, $31^{\circ} 30^{\prime} \mathrm{S}, 51^{\circ} 00^{\prime} \mathrm{W}$, MZSP 27139, 22 m, 13 spm. (R/V "W. Besnard" coll., 25 January 1972). off Rio Grande, $32^{\circ} 13^{\prime} \mathrm{S}, 50^{\circ} 35^{\prime} \mathrm{W}$, MZSP 27136, $84 \mathrm{~m}$, 2 spm. (R/V "W. Besnard" coll., 22 January 1972). 32²0'S, $51^{\circ} 22^{\prime} \mathrm{W}$, MZSP 27128, 52 m, 8 spm. (R/V “W. Besnard" coll., 21 January 1972). $32^{\circ} 28^{\prime} \mathrm{S}, 51^{\circ} 07^{\prime} \mathrm{W}, \mathrm{MZSP} 27155,61 \mathrm{~m}$, 5 spm. (R/V “W. Besnard" coll., 03 November 1972). 32²8'S, $52^{\circ} 15^{\prime} \mathrm{W}$, MZSP 47314, 15 m, 10 spm. (R/V “W. Besnard" coll., 20 January 1972). MZSP 47292, 2 spm. (R/V "W. Besnard" coll., 17 August 1968). MZSP 18579, 3 spm. (R/V"W. Besnard"

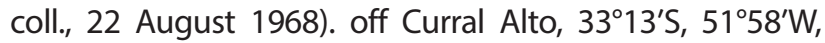
MZSP 27150, 51 m, 2 spm. (R/V "W. Besnard" coll., 02
November 1972). $33^{\circ} 16^{\prime} \mathrm{S}, 52^{\circ} 05^{\prime} \mathrm{W}$, MZSP 18601, 4 spm. (R/V "W. Besnard" coll., 13 December 1968). 3332'S, 52¹8'W, MZSP 18619, 2 spm. (R/V“W. Besnard" coll., 13 March 1969). MZSP 27158, 20 spm. (No additional information).

Distribution: Southwestern Atlantic Ocean; usually from southern Brazil $\left(20^{\circ} \mathrm{S}\right)$ to northern Patagonia, Argentina $\left(46^{\circ} \mathrm{S}\right)$ (Jereb \& Roper, 2010).

\section{Description}

Mantle: Cylindrical and muscular ranging to about $89 \mathrm{~mm}$ (Table 3; Fig. 2A); 3 times longer than wide.

Head: Eye diameter approximately 12\% ML (Table 4). Length and width very similar to each other.

Chromatophores: Distributed throughout body, especially on dorsal region including head (around eyes); dorsal midline of mantle forming an expressive solid line in region free from fins; few chromatophores clustering in middle of fins and at arms/tentacles (Fig. 2A). Mature males do not present stripped chromatophores along ventral portion of mantle.

Fins: Rhomboid, posteriorly connected to mantle, slightly ovate and moderately long, comprising 59\% ML (Table 4; Fig. 2A).

Arms: Muscular, thick at base and distally tapering into slender tips; formula III >IV>II>I ( lengths: $41 \mathrm{~mm}, 38 \mathrm{~mm}, 36 \mathrm{~mm}, 28 \mathrm{~mm}$ ); two rows of suckers. Hectocotylus consists of $\sim 40 \%$ of left ventral arm length extending to arm tip (Table 4). Left row of suckers modified into a simple peduncle whereas right row remaining as suckers. No connection between modified row and suckers, forming valley pattern throughout hectocotylus (Fig. 2E).

Tentacle: Club long and broad, 37\% ML (Table 4). Carpus, manus and dactylus uniformly distributed, with boundaries not very sharp, hindering their definition (especially between manus and dactylus); 4 diagonal rows of suckers along club as stated for D. pleii; 2 central rows at manus not so different in size from other suckers (Fig. 2B). Each sucker with small ring of teeth apparently at surface of sucker's aperture. Longitudinal muscle around suckers present (Fig. 2D).

Circulatory and central nervous system: Similar to preceding species. No apparent morphological differences (Fig. 3A).

Digestive system: Pigmented tip of upper beak is long and slender (Fig. 2C). Remaining structures similar to preceding species.

Male genital system: Similar to preceding species. No apparent morphological differences (Figs. 6A, 6B) except for hectocotylus above described. 
Table 3. Biometric measurements of Doryteuthis sanpaulensis. ML: mantle length (mm); FL: fins length (mm); ED: eye diameter (mm); Al, All, Alll, AIV: arms length dorsally to ventrally $(\mathrm{mm})$; $H \mathrm{HL}$ : hectocotylus length $(\mathrm{mm})$; $\mathrm{CL}$ : tentacular club length $(\mathrm{mm})$.

\begin{tabular}{|c|c|c|c|c|c|c|c|c|c|c|c|c|c|c|c|c|c|c|c|c|c|}
\hline \multicolumn{2}{|c|}{$\begin{array}{c}\text { Individual } \\
\text { Sex }\end{array}$} & $\begin{array}{l}1 \\
\wp\end{array}$ & $\begin{array}{l}2 \\
0^{n}\end{array}$ & $\begin{array}{l}3 \\
0^{7}\end{array}$ & $\begin{array}{l}4 \\
\wp\end{array}$ & $\begin{array}{l}5 \\
q\end{array}$ & $\begin{array}{l}6 \\
\wp\end{array}$ & $\begin{array}{l}7 \\
0^{2}\end{array}$ & $\begin{array}{l}8 \\
0^{7}\end{array}$ & $\begin{array}{l}9 \\
0^{7}\end{array}$ & $\begin{array}{l}10 \\
\wp\end{array}$ & $\begin{array}{l}11 \\
0^{7}\end{array}$ & $\begin{array}{l}12 \\
\wp\end{array}$ & $\begin{array}{l}13 \\
q\end{array}$ & $\begin{array}{c}14 \\
\wp\end{array}$ & $\begin{array}{l}15 \\
\wp\end{array}$ & $\begin{array}{c}16 \\
q\end{array}$ & $\begin{array}{l}17 \\
\wp\end{array}$ & $\begin{array}{l}18 \\
\sigma^{7}\end{array}$ & $\begin{array}{l}19 \\
\sigma^{7}\end{array}$ & $\begin{array}{l}20 \\
0^{7}\end{array}$ \\
\hline \multicolumn{2}{|l|}{ ML } & 92 & 116 & 110 & 92 & 102 & 95 & 94 & 77 & 82 & 74 & 85 & 75 & 70 & 75 & 75 & 70 & 74 & 55 & 145 & 149 \\
\hline \multicolumn{2}{|l|}{$\mathrm{FL}$} & & 70,1 & 64,9 & 53,2 & 63,6 & 56,2 & 56,1 & & 47,3 & 43,4 & & 45,1 & 42,3 & & & 42,6 & 42,1 & & & 90,6 \\
\hline \multicolumn{2}{|l|}{ ED } & 10,9 & 10,7 & 10,9 & 10 & 11,1 & 10,2 & 10,1 & 9,7 & 10,9 & 9,8 & 10,5 & 8,7 & 8,7 & 8,3 & 11 & 11,5 & 8,6 & 8,3 & 2,2 & 12,4 \\
\hline \multirow[t]{6}{*}{ LEFT } & $\mathrm{Al}$ & 31,1 & 33,5 & 30,5 & 29,2 & 37 & 25,6 & 26,7 & 27,8 & 27 & 25 & 29,6 & 25,5 & 22,8 & 26,4 & & 33,3 & & & & 40 \\
\hline & All & 38 & 44, & 40,8 & 38,1 & 49,2 & 37,8 & 38,6 & 4 & 36,5 & 32,2 & 39,2 & 33,4 & 32,9 & 8 & & 29,3 & 27,8 & 24,5 & 6 & 50,3 \\
\hline & Alll & 43,4 & 49,6 & 41,4 & 46,1 & 55,1 & 35,9 & 44,4 & 35,4 & 38,2 & 39 & 40,5 & 38,7 & 35,7 & 37,2 & & 3,5 & 24,8 & & & 55,4 \\
\hline & AIV & 43,1 & 38,2 & 47,3 & 10,3 & 49,2 & 38,7 & 40,8 & & 31,1 & 35,5 & 29,9 & 33,5 & 33,1 & 34,6 & & 49,3 & 30,3 & 24,8 & & 52,9 \\
\hline & HtL & - & 17,8 & 15,8 & - & - & - & 15 & 12,6 & 15,2 & - & 12,8 & - & - & - & - & - & - & 9,9 & 1,3 & 17,8 \\
\hline & $\mathrm{CL}$ & 34,4 & 38,7 & 36 & 33,5 & 43,9 & 30,3 & 32,1 & & & 29,8 & 27 & 35,2 & 30,2 & 30,2 & 32,5 & 34,4 & 27,7 & & & 46,6 \\
\hline \multirow[t]{5}{*}{ RIGHT } & Al & 29,9 & 22,2 & 29,7 & 30,8 & 34,3 & 28,5 & 26,1 & 25,7 & 28,3 & 25,8 & 27,2 & 27,2 & 23 & 25 & & 24 & 21 & 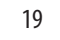 & & 42,5 \\
\hline & All & 38,6 & 44,2 & 32,7 & 39,6 & 50,5 & 34,9 & 38,7 & 32,6 & 37,2 & 32,2 & 38,4 & 32,1 & 34,5 & 32,4 & 41, & 45,4 & 27,8 & 22,2 & 46,4 & 49,5 \\
\hline & AllI & 45,8 & 48,7 & 45,1 & 45,9 & 54,2 & 37,6 & 44,6 & 35,8 & 38,9 & 34,6 & 39,4 & 42,2 & 36 & 9 & & 5,4 & 2 & 4 & ,5 & 53,3 \\
\hline & AIV & 40,3 & 40,1 & 44,9 & 44,2 & 51,4 & 39,6 & 39,4 & 31,8 & 34,5 & 36 & 35,2 & 35 & 33,3 & 34,1 & 47 & 47,1 & 31,1 & 24 & & 55,1 \\
\hline & CL & 19,8 & 34,9 & 35,5 & 33,5 & 46,9 & 31,5 & 32,3 & 23 & 28,5 & 29 & 30,2 & 35,1 & 30,7 & 35,9 & 33 & 32,9 & 29,2 & 22,7 & 38,6 & 48,3 \\
\hline \multicolumn{2}{|c|}{ Individual } & 21 & 22 & 23 & 24 & 25 & 26 & 27 & 28 & 2 & 30 & 31 & 32 & 33 & 34 & 3 & 36 & 37 & & 39 & 40 \\
\hline \multicolumn{2}{|c|}{ Sex } & $0^{x}$ & $0^{x}$ & $q$ & $0^{x}$ & q & 웅 & 웅 & $0^{x}$ & 웅 & $\sigma^{x}$ & 운 & q & $0^{x}$ & $q$ & $0^{*}$ & 웅 & 운 & $0^{x}$ & $0^{\pi}$ & q \\
\hline \multicolumn{2}{|l|}{ ML } & 115 & 107 & 111 & 78,7 & 70 & 71,8 & 69 & 85,3 & 98,2 & 146 & 96,1 & 75,9 & 75,4 & 84,3 & 6 & 75,8 & & & 71,8 & 74 \\
\hline \multicolumn{2}{|l|}{$\mathrm{Fl}$} & 70,4 & 63,2 & 66,2 & 47 & 41,6 & 37,4 & 39 & 5 & $61, \varepsilon$ & 87,2 & & 41, & 42,3 & & 3 & 43 & 9 & 51 & & 43,3 \\
\hline \multicolumn{2}{|l|}{ ED } & 11 & 9,9 & 10,4 & 9,6 & 9,4 & 9,8 & 9,4 & 8,1 & 12,8 & 14 & 13,3 & 10,9 & 10 & 13,9 & 11 & 11,3 & 12,6 & 12,6 & 11 & 11,4 \\
\hline \multirow[t]{6}{*}{ LEFT } & Al & 35,6 & 34,8 & 34,4 & 22,5 & 20,3 & 23,2 & 22,5 & 22,6 & - & 32,6 & 30 & 25,4 & 25,7 & - & 21,5 & 23,3 & 29,6 & & 25 & - \\
\hline & All & 46,6 & 42,8 & 41,8 & 26,3 & 26,8 & 31,9 & 26,8 & 28 & - & 35,8 & 36 & 32,3 & 33,9 & - & 26,4 & 29,1 & 41,8 & 34,7 & 34,3 & - \\
\hline & AllI & 49,6 & 49 & 48,9 & 30,6 & 33 & 35,8 & 33,5 & 32,4 & - & 50,9 & 46,5 & 39 & 36,8 & - & 32 & 33,2 & 44,5 & 36,6 & 8,7 & - \\
\hline & AIV & 48 & 41,8 & 46,8 & 29,7 & 31,7 & 32,4 & 31,9 & 32,3 & - & 51,2 & 45,9 & 38 & 32 & - & 31 & 36,5 & 42 & 35,3 & 32,7 & - \\
\hline & HtL & 19,1 & 16,8 & - & & - & - & - & 12,9 & - & & - & - & 13,9 & - & 14 & - & - & & 11 & - \\
\hline & CL & 34,3 & 41,1 & 41 & 30,8 & 33,6 & 31,2 & 29,7 & 29,8 & 32,1 & 45,9 & 30,6 & 29,4 & 28,8 & 31,5 & 28,7 & 31,1 & 38,4 & 30,6 & 28,9 & 29,9 \\
\hline \multirow[t]{5}{*}{ RIGHT } & Al & 34,5 & 34,1 & 33,7 & 22 & 21 & 22,4 & 22,8 & 22,6 & - & 32,8 & 31,1 & 24,3 & 27,5 & - & 21 & 23,3 & 29,6 & 27,2 & 25 & - \\
\hline & All & 41,2 & 35,5 & 45,3 & 26,1 & 28,4 & 30,8 & 28,9 & 27,9 & - & 35 & 35,9 & 32 & & - & 26 & 3,7 & 41,8 & 34,7 & 34 & - \\
\hline & Alll & 44,7 & 46,7 & 54,2 & 32 & 33,1 & 32,8 & 33,5 & 31,8 & - & 51,4 & 45 & 38,8 & 36,8 & - & 31,8 & 34,4 & 44,5 & 36,5 & 38,6 & - \\
\hline & AIV & 52,5 & 45,7 & 50,6 & 30,7 & 31,3 & 33,1 & 33,3 & 30,3 & - & 56 & 44,8 & 37,6 & 33,6 & - & 29,8 & 33,4 & 42 & 35 & 33,4 & - \\
\hline & CL & 42 & 41,5 & 41,5 & 30,6 & 32,9 & 31,7 & 30,3 & 29,9 & 31,5 & 45,3 & 31,2 & 30,1 & 28,5 & 32 & 29,1 & 32 & 38,3 & 31,2 & 28,8 & 29,8 \\
\hline
\end{tabular}

Table 4. Proportions between characters of Doryteuthis sanpaulensis. FL-ML: Proportion between fin length and mantle length (\%); ED-ML: Proportion between eye diameter and mantle length (\%); HtL-Lft AIV: Proportion between hectocotylized portion and left ventral arm length (\%). CL-ML: Proportion between tentacular club length and mantle length (\%).

\begin{tabular}{|c|c|c|c|c|c|c|c|c|c|c|c|c|c|c|c|c|c|c|c|c|}
\hline & 1 & 2 & 3 & 4 & 5 & 6 & 7 & 8 & 9 & 10 & 11 & 12 & 13 & 14 & 15 & 16 & 17 & 18 & 19 & 20 \\
\hline & ㅇ & $0^{2}$ & $\sigma^{\pi}$ & 우 & 웅 & 우 & $0^{7}$ & $\sigma^{7}$ & $0^{2}$ & 우 & $0^{\pi}$ & 우 & ㅇ & q & q & 우 & 우 & $0^{n}$ & $0^{\prime \prime}$ & $\sigma^{7}$ \\
\hline FL-ML & 59,9 & 60,4 & 59 & 57,8 & 62,4 & 59,2 & 59,7 & 56 & 57,7 & 58,6 & 60,2 & 60,1 & 60,4 & 57,1 & 61,1 & 60,9 & 56,9 & 58,7 & 59,4 & 60,8 \\
\hline ED-ML & 11,8 & 9,22 & 9,91 & 10,9 & 10,9 & 10,7 & 10,7 & 12,6 & 13,3 & 13,2 & 12,4 & 11,6 & 12,4 & 11,1 & 14,7 & 16,4 & 11,6 & 15,1 & 8,41 & 8,32 \\
\hline HtL-Lft AIV & - & 46,6 & 33,4 & - & - & - & 36,8 & 40,1 & 48,9 & - & 42,8 & - & - & - & - & - & - & 39,9 & 47,1 & 33,6 \\
\hline \multirow[t]{3}{*}{ CL-ML } & 29,5 & 31,7 & 32,5 & 36,4 & 44,5 & 32,5 & 34,3 & 29,7 & 34,5 & 39,7 & 33,8 & 46,9 & 43,5 & 44,1 & 43,9 & 48,1 & 38,4 & 40,6 & 26,4 & 31,8 \\
\hline & 21 & 22 & 23 & 24 & 25 & 26 & 27 & 28 & 29 & 30 & 31 & 32 & 33 & 34 & 35 & 36 & 37 & 38 & 39 & 40 \\
\hline & $\sigma^{x}$ & $\sigma^{x}$ & 우 & $\sigma^{2}$ & 우 & 우 & 우 & $0^{x}$ & 우 & $\sigma^{\prime \prime}$ & 우 & 우 & $\sigma^{2}$ & 우 & $0^{x}$ & 우 & 우 & $0^{x}$ & $0^{x}$ & 우 \\
\hline FL-ML & 61,2 & 59,1 & 59,6 & 59,7 & 59,4 & 52,1 & 56,5 & 58,6 & 62,9 & 59,8 & 57,2 & 54,9 & 56,1 & 61,7 & 58,1 & 56,7 & 60,2 & 61,3 & 59,9 & 58,5 \\
\hline ED-ML & 9,57 & 9,25 & 9,37 & 12,2 & 13,4 & 13,6 & 13,6 & 9,5 & 13 & 9,6 & 13,8 & 14,4 & 13,3 & 16,5 & 17,7 & 14,9 & 12,9 & 14,9 & 15,3 & 15,4 \\
\hline HtL-Lft AIV & 39,8 & 40,2 & - & 37,4 & - & - & - & 39,9 & - & 38,1 & - & - & 43,4 & - & 45,2 & - & - & 34,8 & 33,6 & - \\
\hline CL-ML & 33,2 & 38,6 & 37,2 & 39 & 47,5 & 43,8 & 43,5 & 35 & 32,4 & 31,3 & 32,2 & 39,2 & 38 & 37,7 & 46,6 & 41,6 & 39,1 & 36,7 & 40,2 & 40,3 \\
\hline
\end{tabular}

Female genital system: Similar to preceding species except for nidamental glands with prominent thin tip (Fig. 5D).

\section{DISCUSSION}

Doryteuthis pleii and $D$. sanpaulensis are really similar to each other, sometimes difficult to separate in the field and in preserved samples. The main differences between them resides in the gladial features and in external proportions (Juanicó, 1979; Brakoniecki, 1984). However, the overall anatomy of both species described herein showed additional distinguishing characters. The tentacular club of $D$. pleii has two central rows of suckers larger than the marginal ones (Fig. 1B), whereas D. sanpaulensis has four similarly sized rows of suckers (Fig. 2B), where the marginal rows are just slightly smaller than the central ones. 


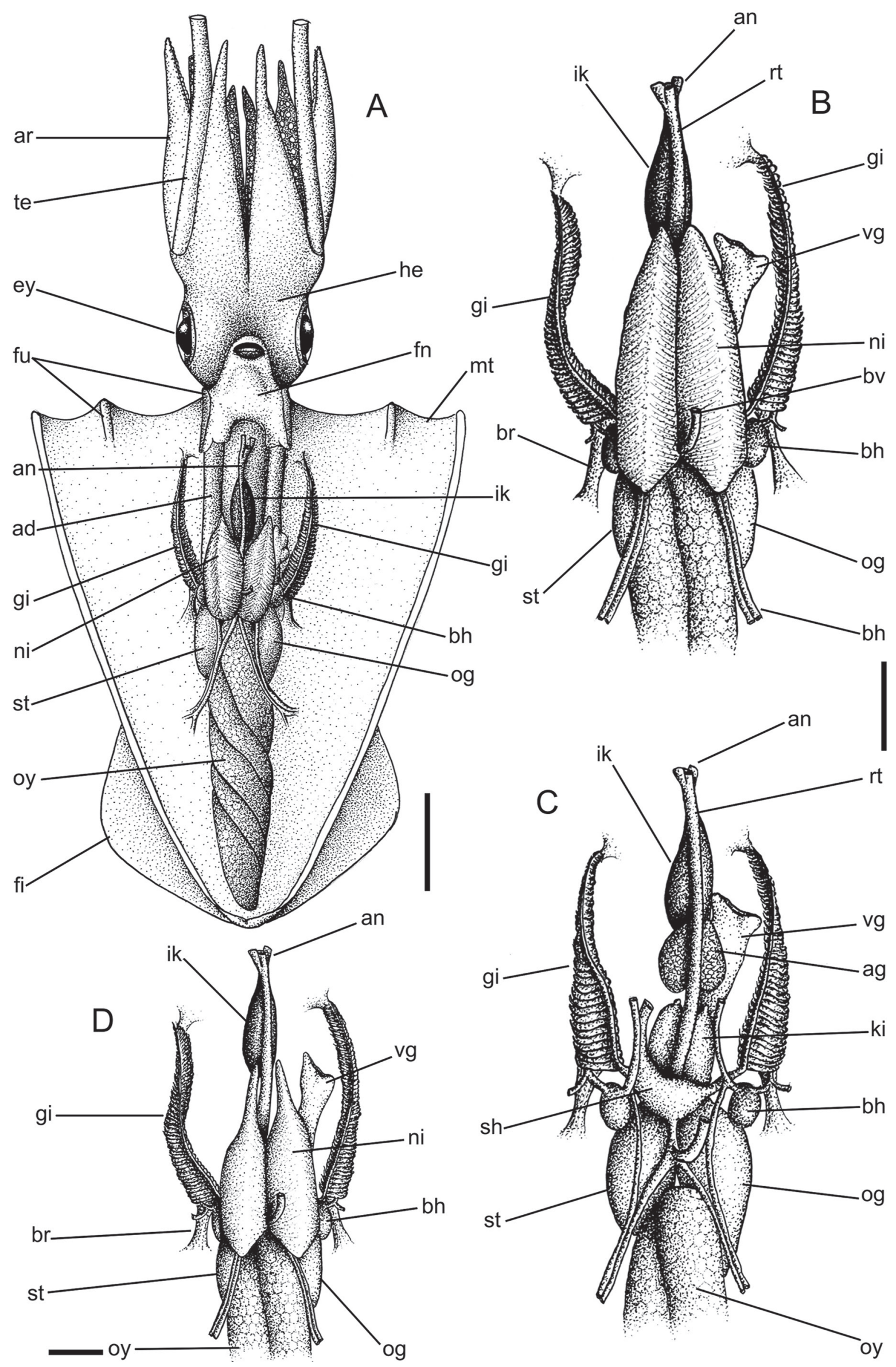

Figure 5. (A) Female, representing both species, ventral view, mantle opened longitudinally, scale $=20 \mathrm{~mm}$; (B) Doryteuthis pleii female reproductive system, ventral view, scale $=10 \mathrm{~mm}$; (C) Female reproductive system representing both species nidamental gland removed, scale $=10 \mathrm{~mm}$; (D) Doryteuthis sanpaulensis female reproductive system, ventral view, scale $=10 \mathrm{~mm}$. 
Furthermore, the relation between the club length and mantle length is different between these species. For D. pleii, the ratio is around $28 \%$ of mantle length, which was also found by Cohen (1976), who stated that the club of D. pleii is $19-29 \%$ of ML. While for D. sanpaulensis, the ratio is around $37 \%$, which is larger possibly due to the similar size of the suckers along the club, which increases its overall size.
The mantle itself does not have large differences between these species. However, the mantle-fin ratio in D. pleii usually reaches approximately $40-50 \%$, hardly no more than $1 / 2$ of mantle length (Fig. $1 A$ ), while in $D$. sanpaulensis this ratio usually stays around $60 \%$ or more (Fig. 2A). Additionally, Brakoniecki (1984) stated that the mantle-fin ratio of $D$. pleii are more similar to D. gahi than to $D$. sanpaulensis.

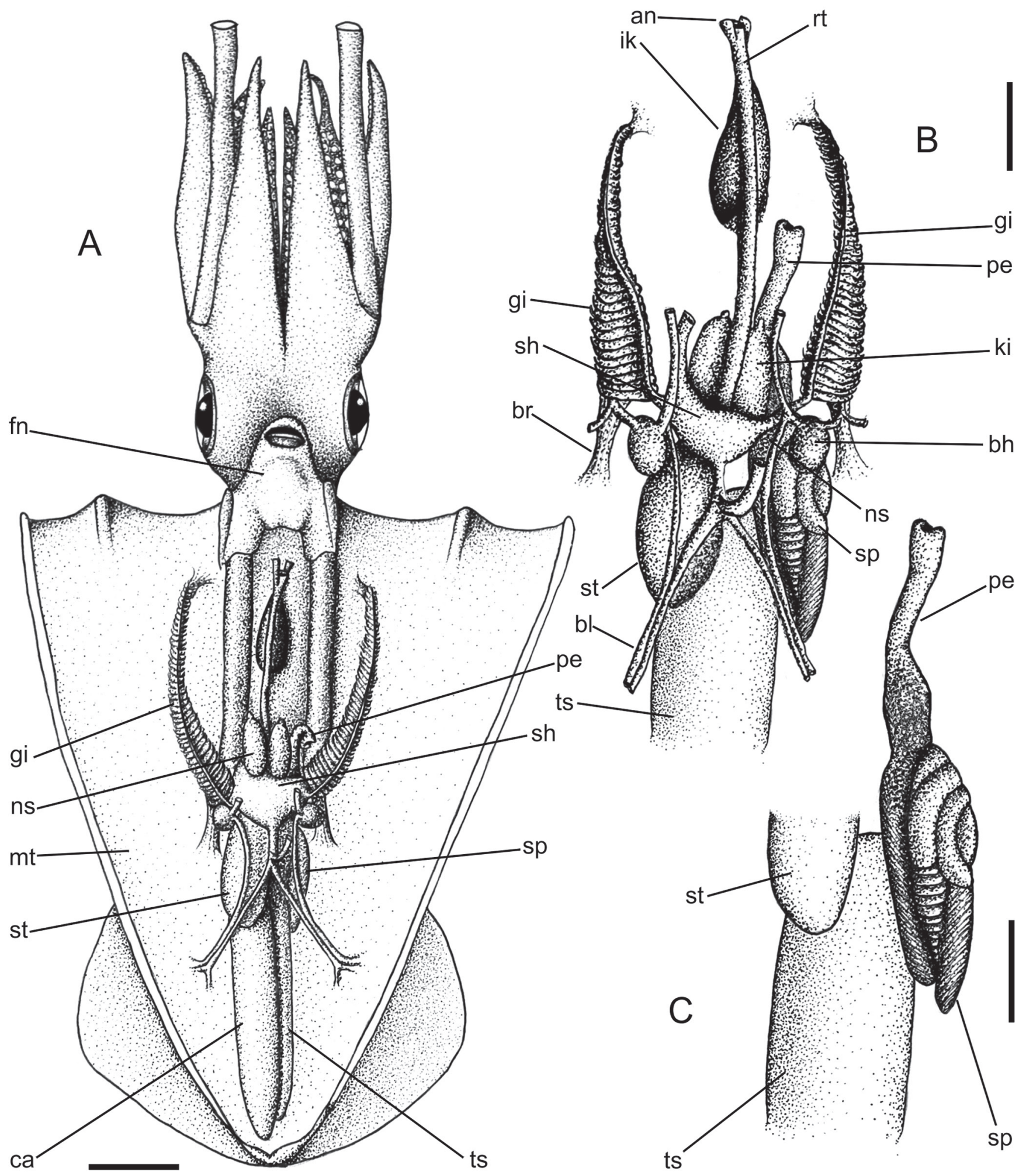

Figure 6. (A) Male, representing both species, ventral view, mantle opened longitudinally, scale $=20 \mathrm{~mm}$; (B) Detail of male reproductive system representing both species, ventral view, scale $=10 \mathrm{~mm}$; (C) Male reproductive system isolated, representing both species, ventral view, scale $=10 \mathrm{~mm}$. 
Cohen (1976) stated that the hectocotylized portion of D. pleii is no more than $1 / 2$ of the left ventral arm length, which was confirmed here. The hectocotylized portion is practically the same for these two specimens, comprising 39-40\% of the left ventral arm length. However, the morphology of the hectocotylus differs between them. Doryteuthis pleii shows a bar connecting the modified and unmodified row, forming a ladder pattern (Fig. 1E), whilst D. sanpaulensis does not have any kind of structure connecting the rows, forming a valley between the rows (Fig. 2E).

The eye diameter of $D$. pleii has been referred as about 14-19\% of mantle length (Brakoniecki, 1984); however, this study has obtained the eye diameter of around $13 \%$. This difference has been interpreted as a variation in the southern population. As evidenced herein, the eye diameter in D. sanpaulensis is around $12 \% \mathrm{ML}$.

The statistical morphometric analyses done by Juanicó (1979) already showed the distinction between both species, including ontogenetic and dimorphism variation. The preliminary results of the data of the present paper greatly coincided with those of Juanicó (1979), which was also partially included in Perez et al. (2002), dispensing the need of additional analyses. This paper remains, then, focused on the visible morpho-anatomical features of adult specimens.

The internal anatomy does not show significant differences between these two species. The circulatory and the nervous systems have practically the same features in the two species (Fig. 3). Regarding the digestive system, only the beak structure has shown some differences. The pigmented tip of the upper beak of $D$. pleii is short and robust (Fig. 1C), whereas that of $D$. sanpaulensis has a long and slender tip (Fig. 2C). Moreover, the radulae also did not have expressive differences to distinguish the species (Fig. 4). Doryteuthis pleii and D. sanpaulensis have typical squid radulae: rachidian and first lateral teeth with a primary large projection and secondary smaller one(s) (Fig. 4).

As for the genital system, the nidamental glands are more uniform in $D$. pleii females, without a slender tip (Fig. 5B), whilst in D. sanpaulensis females, the nidamental glands have a more prominent, thin anterior tip (Fig. 5D). On the other hand, there were no significant differences between the male genital system of both species (Fig. 6). In the case of the male genital system, spermatophores are good sources of distinguishing characters, since they are highly complex and a key structure to differentiate species (Marian \& Domaneschi, 2012; Marian, 2012).

The genus Doryteuthis is divided into three subgenera: (i) Amerigo (Brakoniecki, 1986) that comprises D. gahi (D'Orbigny, 1835), D. ocula (Cohen, 1976), D. opalescens (Berry, 1911), D. pealeii (Lesueur, 1821) and D. surinamensis (Voss, 1974), (ii) Doryteuthis s.s. (Naef, 1912), encompassing D. pleii (type species) and D. roperi (Cohen, 1976) and (iii) the undescribed subgenus of $D$. sanpaulensis (sensu Vecchione et al., 2005; Jereb \& Roper, 2010). Amerigo and Doryteuthis are separated by differences in the gladial structure and in the hectocotylized portion in the left ventral arm (Jereb \& Roper, 2010; Vecchione \& Young, 2010b).

Even though there are interesting characters listed in this study that can be used to further distinguish $D$. pleii from $D$. sanpaulensis, there are also some characteristics that might bring them phylogenetically closer. This is the case of the size of hatchlings and eggs (Barón, 2003b); the slightly exclusive internal anatomy; and the morphology of the hectocotylus [Brakoniecki (1986) once clustered D. pleii and D. sanpaulensis together using this character]. The key to the subgenus Doryteuthis, as proposed by Vecchione et al. (2005), is based on the hectocotylized portion extending to the arm tip, and on the thickened gladius vane for Doryteuthis; a characters absent in Amerigo; for D. sanpaulensis, the hectocotylized portion is similar to those in the Doryteuthis s.s. Therefore, the undescribed subgenus of $D$. sanpaulensis might be phylogenetically closer to Doryteuthis than to Amerigo. Certainly, more studies are required to clarify this relation among Doryteuthis species as well as a complete study to describe the subgenus of $D$. sanpaulensis, or to consider it definitively as Doryteuthis.

As the separation between Loligo and Doryteuthis has been essentially based on molecular results, another intention of this paper is furnishing additional, morphological subsides for genus' definition. Possibly further studies on more species of these two taxa in the same level as described here can supply identification by simply inspection, dispensing laboratorial procedures or morphometric analyses. On the other hand, it is recognized that the relative uniformity of the internal anatomy of both species herein studied cannot resist to further investigation, such as more details of the digestive system (e.g., odontophore and beak muscles), of the brain, etc. These details are still being developed, and are not the present scope.

\section{CONCLUSIONS}

1) Doryteuthis pleii and D. sanpaulensis are really close taxa, and possibly belong to the same subgenus (Doryteuthis s.s.).

2) Despite their similarities, at least details and proportions of the mantle color and form, mantle-fin ratio, tentacular club, hectocotylus, gladius, and eye, can provide relative easy identification between there both sympatric species.

3) The internal anatomy, in the present level of details, is relatively uniform between both species; significant differences are fond in beak and nidamental glands.

\section{ACKNOWLEDGEMENTS}

We thank the Swansea University, especially Dr. John Griffin for his helpful suggestions; Brazilian Government Program Science Without Borders and the Conselho Nacional de Desenvolvimento Científico e Tecnológico (CNPq), which provided support throughout the year abroad to PPM; Fundação de Amparo à Pesquisa do Estado de São Paulo (FAPESP); Claudia Guimarães, Jaime Jardim, Daniel Cavallari, Patricia Lima and Mariana Mendes for many helpful comments along the project. 


\section{REFERENCES}

Allcock, A.L.; Lindgren, A. \& Strugnell, J. 2015. The contribution of molecular data to our understanding of cephalopod evolution and systematics: a review. Journal of Natural History, 49(21-24): 1373-1421.

Anderson, F.E. 2000. Phylogenetic relationships among loliginid squids (Cephalopoda: Myopsida) based on analyses of multiple data sets. Zoological Journal of the Linnean Society, 130(4): 603-633.

Andriguetto, J.\&Haimovici, M. 1996. Reproductive cycle of Loligo sanpaulensis Brakoniecki, 1984 (Cephalopoda; Loliginidae) in southern Brazil. Scientia Marina, 60(4): 443-450.

Barcellos, D.D. \& Gasalla, M.A. 2014. Morphology and morphometry of Doryteuthis plei (Cephalopoda: Loliginidae) statoliths from the northern shelf off São Paulo, southeastern Brazil. Journal of Natural History, 49(21-24): 1305-1317.

Barón, P.J. 2003a. Embryonic development of the South American long-fin squid Loligo sanpaulensis Brakoniecki, 1984. Journal of Molluscan Studies, 69(3): 221-227.

Barón, P.J. 2003b. The paralarvae of two South American sympatric squid: Loligo gahi and Loligo sanpaulensis. Journal of Plankton Research, 25(11): 1347-1358.

Barón, P.J. \& Ré, M.E. 2002. Morphometry of the northern Patagonian sympatric populations of Loligo sanpaulensis and Loligo gahi. Journal of the Marine Biological Association of the United Kingdom, 82(2): 269-278.

Brakoniecki, T.F. 1984. A full description of Loligo sanpaulensis, Brakoniecki, 1984 and a redescription of Loligo gahi d'Orbigny, 1835, two species of squid (Cephalopoda; Myopsida) from the Southwest Atlantic. Bulletin of Marine Science, 34(3): 435-448.

Brakoniecki, T.F. 1986. A generic revision of the family Loliginidae (Cephalopoda; Myopsida) based primarily on the comparative morphology of the hectocotylus (zoogeography). PhD Thesis, University of Miami, Coral Gables.

Brakoniecki, T.F. 1996. A revision of the genus Pickfordiateuthis Voss, 1953 (Cephalopoda; Myopsida). Bulletin of Marine Science, 58: 9-28.

Cardoso, F.; Baltazar, P. \& Bautista, J. 2005. The early development of the Patagonian squid Loligo gahi d'Orbigny, 1835 in Peruvian Waters (Cephalopoda: Loliginidae). Revista Peruana de Biología, 12(3): 369-376.

Cohen, A.C. 1976. The systematics and distribution of Loligo (Cephalopoda, Myopsida) in the western North Atlantic, with descriptions of two new species. Malacologia, 15(2): 299-367.

Costa, P.A.S. \& Fernandes, F.d.C. 1993. Seasonal and spatial changes of cephalopods caught in the Cabo Frio (Brazil) upwelling ecosystem. Bulletin of Marine Science, 522): 751-759.

Couto, D.R.; Simone, L.R.L. \& Pimenta, A.D. 2015. Comparative anatomy of the fasciolariids Pustulatirus ogum and Hemipolygona beckyae from Brazil (Gastropoda: Buccinoidea: Peristerniinae). Scientia Marina, 79(1): 89-105.

Crespi-Abril, A.; Gomila, G.V.; Venerus, L. \& Barón, P. 2014. Spatial distribution of cephalopod paralarvae in San José Gulf (Northern Patagonia, Argentina): the role of tidal circulation in larval dispersal. Fisheries Research, 152: 13-20.

de Aguiar, D.C.; Rossi-Wongtschow, C.L.D.B. \& Perez, J.A.A. 2012. Validation of daily growth increments of statoliths of Brazilian squid Doryteuthis plei and D. sanpaulensis (Cephalopoda: Loliginidae). Bioikos, 26(1): 13-21.

de Luna Sales, J.B.; Shaw, P.W.; Haimovici, M.; Markaida, U.; Cunha, D.B.; Ready, J.; Figueiredo-Ready, W.M.; Schneider, H. \& Sampaio, I. 2013. New molecular phylogeny of the squids of the family Loliginidae with emphasis on the genus Doryteuthis Naef, 1912: Mitochondrial and nuclear sequences indicate the presence of cryptic species in the southern Atlantic Ocean. Molecular Phylogenetics and Evolution, 68(2): 293-299. de Pasquier, G.A. \& Carroz, S.R. 2012. El calamar flecha, Doryteuthis (Doryteuthis) plei (Blainville, 1823), en la bahía el tablazo, Venezuela. Boletín del Centro de Investigaciones Biológicas, 46(1): 33-43.

DiMarco, F.P. \& Hanlon, R.T. 1997. Agonistic behavior in the squid Loligo plei (Loliginidae, Teuthoidea): fighting tactics and the effects of size and resource value. Ethology, 103(2): 89-108.

Dornellas, A.P.S. \& Simone, L.R.L. 2015. New morphological data on Solariella obscura (Trochoidea: Solariellidae) from New Jersey, USA. Zoologia, 32(2): 162-170.

Fields, W.G. 1965. The structure, development, food relations, reproduction and life history of the squid Loligo opalescens Berry. California Fish and Game Bulletin, 131: 1-108.

Granados-Amores, J.; García-Rodríguez, F.J.; Hochberg, F. \& Salinas-Zavala, C.A. 2014. The taxonomy and morphometry of squids in the family Loliginidae (Cephalopoda: Myopsida) from the Pacific coast of Mexico. American Malacological Bulletin, 32(2): 198-208.

Grassi, R.T.B.; dos Santos, R.A.; von Seckendorff, R.W.; Júnior, J.M.; da Camara, J.J.C. \& Campos, E.C. 1990. Variação sazonal na captura de lula (Doryteuthis piei Blainville, 1823) no sudeste do Brasil, utilizando atração luminosa e rede corrediça. Brazilian Journal of Veterinary Research and Animal Science, 27(2): 259-265.

Haimovici, M. \& Andriguetto, J.M. 1986. Cefalópodes costeiros capturados na pesca de arrasto do litoral sul do Brasil. Arquivos de Biologia e Tecnologia, 29(3): 473-495.

Haimovici, M. \& Perez, J.A. 1991. Coastal cephalopod fauna of southern Brazil. Bulletin of Marine Science, 49(1-2): 221-230.

Hanlon, R.T.; Hixon, R.F. \& Hulet, W.H. 1983. Survival, growth, and behavior of the Ioliginid squids Loligo plei, Loligo pealei, and Lolliguncula brevis (Mollusca: Cephalopoda) in closed sea water systems. Biological Bulletin 165: 637-685.

Herke, S. \& Foltz, D. 2002. Phylogeography of two squid (Loligo pealei and L. plei) in the Gulf of Mexico and northwestern Atlantic Ocean. Marine Biology, 140(1): 103-115.

Jackson, G. \& Forsythe, J. 2002. Statolith age validation and growth of Loligo plei (Cephalopoda: Loliginidae) in the north-west Gulf of Mexico during spring/summer. Journal of the Marine Biological Association of the United Kingdom, 82(4): 677-678.

Jereb, P. \& Roper, C. 2010. Cephalopods of the world. An annotated and illustrated catalogue of cephalopod species known to date, vol. 2. Myopsid and Oegopsid Squids. Rome, FA0. 605p. (FAO Species Catalogue for Fishery Purposes, n. 4, v.2).

Juanicó, R.M. 1979. Contribuição ao estudo da biologia dos Cephalopoda Loliginidae do Atlântico Sul Ocidental, entre o Rio de Janeiro e Mar del Plata. PhD Thesis, Instituto Oceanográfico, Universidade de São Paulo, $102 p$.

Laptikhovsky, V.; Arkhipkin, A. \& Bolstad, K. 2009. A second species of the squid genus Kondakovia (Cephalopoda: Onychoteuthidae) from the subAntarctic. Polar Biology, 32: 21-26.

Marian, J.E.A. 2010. Perforating potential of Ioliginid spermatophores. Journal of Molluscan Studies, 77(1):98-100.

Marian, J.E.A. 2012. Spermatophoric reaction reappraised: novel insights into the functioning of the loliginid spermatophore based on Doryteuthis plei (Mollusca: Cephalopoda). Journal of Morphology, 273(3): 248-278.

Marian, J.E. \& Domaneschi, 0. 2012. Unraveling the structure of squids' spermatophores: a combined approach based on Doryteuthis plei (Blainville, 1823) (Cephalopoda: Loliginidae). Acta Zoologica, 93(3): 281-307.

Martins, R.S. \& Perez, J.A.A. 2006. Occurrence of loliginid paralarvae around Santa Catarina Island, southern Brazil. Pan-American Journal of Aquatic Sciences, 1: 24-27. 
Naef, A. 1912. Teuthologische Notizen. 4. Die Gattungen der Loliginidae. Zoologischer Anzeiger, 39: 741-745.

Perez, J.A.A.; Aguiar, D.C.d. \& Santos, J.A.T.d. 2006. Gladius and statolith as tools for age and growth studies of the squid Loligo plei (Teuthida: Loliginidae) off southern Brazil. Brazilian Archives of Biology and Technology, 49(5): 747-755.

Perez, J.A.A.; de Aguiar, D.C. \& Oliveira, U.C. 2002. Biology and population dynamics of the long-finned squid Loligo plei (Cephalopoda: Loliginidae) in southern Brazilian waters. Fisheries Research, 58(3): 267-279.

Pineda, S.; Aubone, A. \& Brunetti, N. 1996. Identificación y morfometría de las mandibulas de Loligo gahi y Loligo sanpaulensis (Cephalopoda, Loliginidae) del Atlántico Sudoccidental. OceanDocs https://www. oceandocs.org/handle/1834/1961. Access in: 06/2018.

Pineda, S.E.; Hernandez, D.R.; Brunetti, N.E. \& Jerez, B. 2002. Morphological identification of two southwest Atlantic loliginid squids: Loligo gahi and Loligo sanpaulensis. OceanDocs https://www.oceandocs.org/ handle/1834/1583. Access in: 06/2018.

Rodrigues, A.R. \& Gasalla, M.A. 2008. Spatial and temporal patterns in size and maturation of Loligo plei and Loligo sanpaulensis (Cephalopoda: Loliginidae) in southeastern Brazilian waters, between $23 \mathrm{~S}$ and $27 \mathrm{~S}$. Scientia Marina, 72(4): 631-643.

Roper, C.F.E. \& Voss, G.L. 1983. Guidelines for taxonomic descriptions of cephalopod species. Memoirs of the National Museum of Victoria, 44: 49-63.

Roper, C.F.E.; Sweeney, M.J. \& Nauen, C.E. 1984. FA0 species catalogue. Cephalopods of the world, an anoted and illustrated catalogue of species of interest to fisheries. FAO Fisheries Synopsis, 3(125): 1-277.

Sanchez, G.; Perry, H.; Trigg, C. \& Vecchione, M. 1996. Morphometry of juvenile and subadult Loligo pealei and L. plei from the northern Gulf of Mexico. Fishery Bulletin, 94(3): 535.
Simone, L.R.L. 2011. Phylogeny of the Caenogastropoda (Mollusca), based on comparative morphology. Arquivos de Zoologia, São Paulo, 42: 161-323.

Vecchione, M. \& Young, R.E. 2010a. Loliginidae Lesueur, 1821. Version 02 September 2010. http://tolweb.org/Loliginidae/19422/2010.09.02 in The Tree of Life Web Project, http://tolweb.org. Access in: 06/2018.

Vecchione, M. \& Young, R.E. 2010b. Doryteuthis Naef, 1912. Version 02 September 2010 (under construction). http://tolweb.org/ Doryteuthis/23877/2010.09.02 in The Tree of Life Web Project, http:// tolweb.org. Access in: 06/2018.

Vecchione, M.; Brakoniecki, T.F.; Natsukari, Y. \& Hanlon, R.T. 1998. A provisional generic classification of the family Loliginidae. Smithsonian Contributions to Zoology, 586: 215-222.

Vecchione, M.; Shea, E.; Bussarawit, S.; Anderson, F.; Alexeyev, D.; Lu, C.C.; Okutani, T.; Roeleveld, M.; Chotiyaputta, C. \& Roper, C.F.E. 2005. Systematics of Indo-West Pacific Loliginids. Phuket Marine Biological Center Research Bulletin, 66: 23-26.

Vecchione, M.; Sosnowski, A. \& Young, R.E. 2015. Walvisteuthis jeremiahi n. sp. (Mollusca: Cephalopoda), an onychoteuthid squid from the Gulf of Mexico. Proceedings of the Biological Society of Washington, 128: 164-175.

Vidal, E.A.; Haimovici, M. \& Hackbart, V.C. 2010. Distribution of paralarvae and small juvenile cephalopods in relation to primary production in an upwelling area off southern Brazil. ICES Journal of Marine Science: Journal du Conseil, 67(7): 1346-1352.

Whitaker, J.D. 1980. Squid catches resulting from trawl surveys off the southeastern United States. Marine Fisheries Review, 42: 39-43.

Willems, T.; De Backer, A.; You, K.W.T.; Vincx, M. \& Hostens, K. 2015. Spatiotemporal distribution patterns of the epibenthic community in the coastal waters of Suriname. Continental Shelf Research, 108: 25-40. 